\title{
VIDEO CORRECTIONS OF UNDERGRADUATE TEACHING LAB REPORTS
}

\author{
Jorge García-Cañadas \\ Department of Industrial Systems Engineering and Design, Universitat Jaume I (SPAIN)
}

\begin{abstract}
Here it is presented a new methodology based on using video recordings for the evaluation and marking of lab reports in the undergraduate teaching labs. Students are typically requested these reports at each lab session. The reports are partly (or fully) used for the determination of the students mark related to the teaching labs. These reports do not always have the quality expected, since undergraduate students frequently experience different difficulties. Video recordings of the marking process of the reports, with the inclusion of a detailed identification and explanation of the mistakes found, have been performed for the Materials Technology subject in the 4th course of the Industrial Technology Engineering degree. These video recordings, unlike the typically adopted corrections using text comments, were more warmly welcomed by the students, increased the comprehension of the mistakes they performed, and helped them to learn how to prepare higher quality reports. In addition, most of the students considered that these video corrections should be generally implemented in all the teaching labs. Finally, it was also found that marking through this method saves a significant amount of marking time to the lecturer.
\end{abstract}

Keywords: Video recordings, video evaluation, marking, lab reports, undergraduate.

\section{INTRODUCTION}

Undergraduate students evaluation is very frequently performed by correcting tasks which are given to the students (exams, reports, oral presentations, etc.) [1]. A key part for the learning process of the students is the clear identification of the mistakes and errors performed in these tasks, which will solve erroneous concepts and will avoid future repetition of these mistakes. This implies for the lecturers to provide a detailed marking of the tasks delivered by the students, which has to be given to them before the next similar task is requested, otherwise the errors found in the previous tasks will continue [2].

In undergraduate teaching labs, students are typically asked to deliver a report of each lab session performed. This report many times follows the typical structure of a research report or article and it is formed by an introduction, the experimental part, the results and discussion, the conclusions, and finally the references. Undergraduate students frequently fail to provide good quality reports [3], due to different difficulties that they experience, such as the clear identification of the content that should go in each of these sections, the suitable report of the obtained results using tables or graphs, the adequate discussion of the results, and the identification of the main conclusions. In order for them to learn properly how to prepare a high quality report, it is very important to provide a detailed correction of the reports delivered by the lecturer, and hence guarantee that the students have a clear knowledge of the mistakes performed before performing the next report.

In order to improve the evaluation process of these reports and the students skills in the preparation of scientific reports, I have performed video corrections of the reports delivered at each teaching lab session by undergraduate students of the Materials Technology subject in the 4th course of the Industrial Technology Engineering degree. I recorded the computer's screen and my voice while detecting the errors in the students reports and doing the marking. Complementary, I used different supporting media, such as lecture notes or images from the internet to improve the explanations. These video corrections had a remarkable acceptance within the students and provided them a more visual and friendly feedback of the errors performed.

\section{METHODOLOGY}

The evaluation of most of the teaching labs from Materials Science subjects of Engineering degrees in our university are performed by marking the students reports, typically one of these reports is delivered by a group of students per each lab session. Consequently, a video correction was 
performed per each lab session. The video correction consisted in recording the computer's screen and the voice of the lecturer while the report is being marked. I used a screen casting software (Screencastify [4], free of charge for $<10$ min videos), which also allowed the direct uploading of the recorded videos to Google Drive immediately after the recording, which facilitates their later sharing with the students. A screenshot from one of the recorded videos is shown in Figure 1. The video corrections were brief and kept at no longer than $10 \mathrm{~min}$, otherwise their visualization could result boring and not very attractive. To fulfil this, the computer's screen is only recorded when an error or comment is made. To facilitate the pause and resume of the recording it is beneficial to define a keyboard shortcut (e.g. Ctrl $+s$ ) so there is no need to use the mouse (which is being recorded) for this, and improves the pace of the recording.

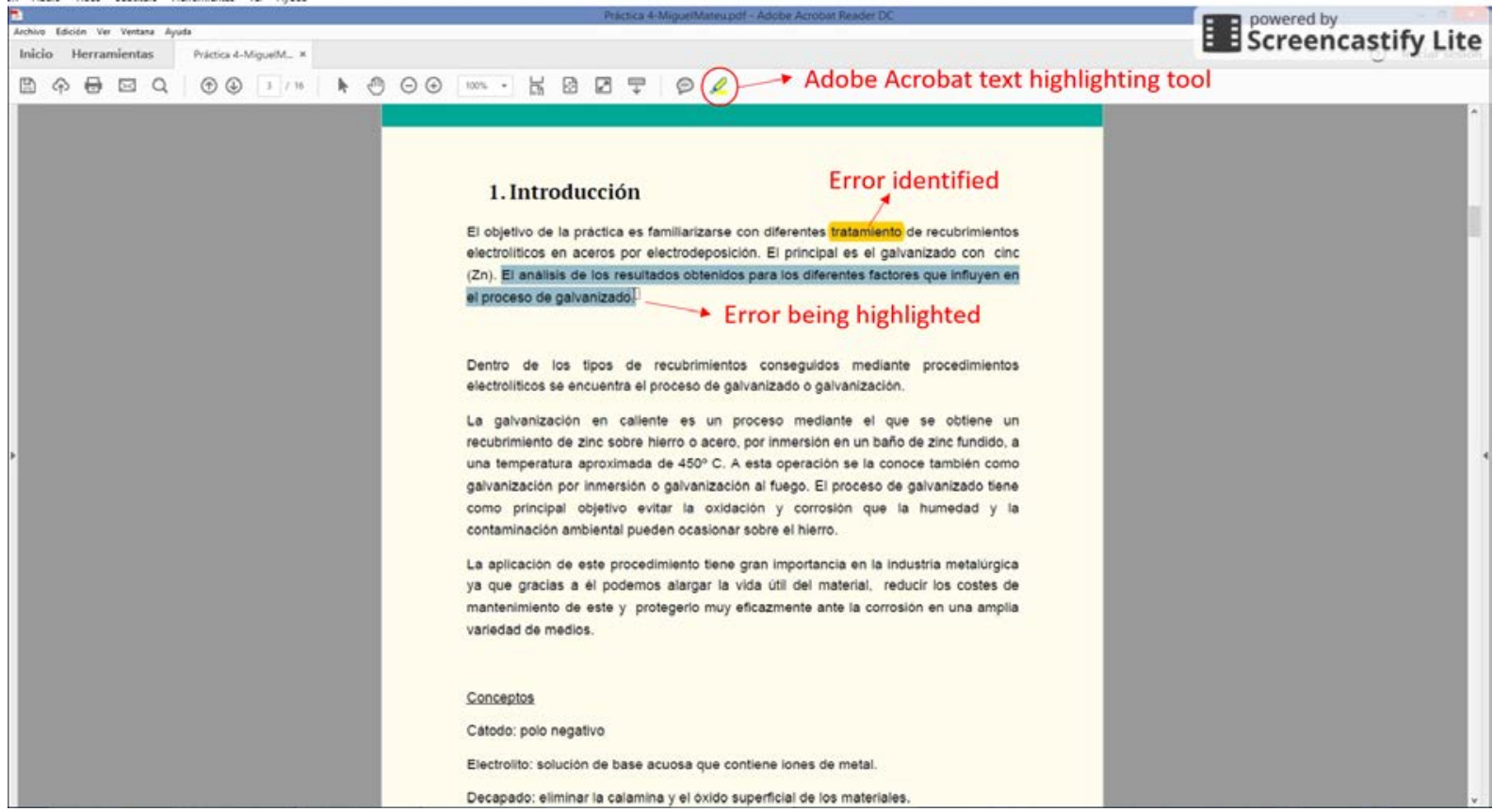

Figure 1. Screenshot of the video correction of a report (in Spanish). The tools used to highlight text and several errors identified are shown.

In order to be able to identify the errors and highlight text, the Acrobat Reader tools can be used (see Figure 1), since the most common format the reports are received is .pdf. On the other hand, many screen casting software also has more advanced highlighting tools or it might exist another software already installed in the computer for this.

An advantage of the video recordings with respect to the typical .pdf annotations is that when mistakes that need a further explanation are identified, this can be performed using additional supporting media that can be easily shown in the screen, such as notes from the theoretical explanations of the subject, examples of high quality reports, websites, additional internet content, etc.

The most common errors that were identified and highlighted in the recordings were:

1 Grammatical and orthographical errors.

2 Contents not properly included in the different sections, such as results being presented in the experimental part.

3 Incorrect representation of results, such as lack of axis titles, wrong units, unclear legends, etc.

4 Wrong calculations.

5 Unreported error bars and standard deviations.

6 Wrong identification of conclusions.

7 Incomplete discussion of the results.

Once the correction of a section is completed, the corresponding mark of this section is typed in a separate document (see Figure 2) that it is also shown in the recording. In this way, it is also very clear 
for the student how his/her final mark is obtained. Moreover, they can also know which sections are weaker and need to be reinforced. When the video correction is finished, the document with the marking, which should also include the link to the video recording (see Figure 2), is sent to the student for his/her visualization.

Your lab report has been marked. The correction of the report can be seen in this video:

https://drive.google.com/file/d/????????

This is how the mark has been obtained:

a) General aspects: 0.7 / 1 .

b) Introduction: 0.5 / 1 .

c) Experimental part: $1.5 / 2$.

d) Results: 1.2 / 2 .

e) Discussion: 1.5 / 2.

f) Conclusions: 2/2.

Total mark: 7.4 / 10

Figure 2. Marking report that includes the different contributions (a to f) to the total mark. This document is completed during the recording and submitted to the student once completed.

\section{RESULTS}

Moving from corrections performed by annotated text in the lab session reports to video corrections has the benefit to save marking time to the lecturer. The first or second initial video corrections might be somewhat lengthy, but once you properly configure the screen casting software and adapt to the recording dynamics, the time saved is significant, and can even reach a $40 \%$ reduction, since you do not need to type the comments or explanations, they are given out loud.

Regarding the impact on the students, it was perceived, specially the first time they receive the video correction, an emotional surprise and a very warm welcome, which is due to the originality of the methodology and the fascination they feel towards videos. Apart from these in-lab perceptions, a survey was performed during the last lab session to collect the students' opinions related to this new methodology. All the students replied that the corrections of the reports were adequate, with all the mistakes identified and remarked, which helped them to understand these errors and avoid their repetition. No other reply apart from that was given.

On the other hand, two more questions were asked, as shown in Table 1. In the first of these questions, $55 \%$ of the students considered that the video corrections were one of the things that they like most from the lab sessions, slightly behind the option with the higher percentage $(64 \%)$ that corresponds to the possibility to handle and measure with different apparatuses. This proves the high degree of acceptance and enjoyment of the video corrections.

Regarding the second question where the students were asked to give their opinion about the use of videos for the marking of the reports (see Table 1), 100\% of the students considered that the video corrections were brilliant and provided a clearer and enjoyable way to know their mistakes, which is in agreement with the above conclusion. On the other hand, $90 \%$ of the students considered that the highlighting of the errors in the video corrections helped them to improve their report writing skills (see Table 1). In addition, $70 \%$ of the students underlined that video corrections should be generally adopted in all the subjects involving lab sessions. Finally, a smaller amount of students $(20 \%)$ considered that the use of video corrections enhanced their motivation (see Table 1). It should be also noted that none of the students considered that just having the final mark of the report or an annotated .pdf was the same than the video marking. 
Table 1. Percentage of students that opted for the different options given to several questions from a survey performed to the students. More than one option was allowed.

\begin{tabular}{l|c}
\hline \multicolumn{1}{c}{ Questions and answers } & Percentage (\%) \\
\hline \multicolumn{1}{c}{ 1. What I like most from the lab sessions is... } & 64 \\
\hline a) To measure and handle the apparatuses. & 27 \\
\hline b) The experimental validation of the theory. & 27 \\
\hline c) When examples or similes from daily life are used in the explanations. & 55 \\
\hline d) The video corrections of the reports. & \\
\hline 2. What is your opinion about the marking of the reports using video corrections? & 0 \\
\hline a) For me it is the same than just providing a number with the final mark. & 100 \\
\hline b) It is brilliant, since I can more clearly know the mistakes made and in a more enjoyable \\
way. & 90 \\
\hline c) The fact of highlighting the mistakes made helps me to learn how to improve the reports. & 70 \\
\hline d) It should be adopted in all the subjects involving lab sessions. & 20 \\
\hline e) It motivates me to learn the subject. & 0 \\
\hline f) For me it would have been the same to have an annotated .pdf instead of the video & \\
\hline \hline
\end{tabular}

All these results clearly prove that video corrections of teaching lab sessions are an excellent way to provide feedback to the students, since it produces a more enjoyable experience, contribute to improve the learning process, and in some cases can also increase the students motivation. It should be also mentioned that this methodology can also be extended to other areas apart from teaching labs. For example, I also used it in the correction of scientific papers prepared by PhD students, experiencing a similar level of acceptance and impact in the learning process of writing research articles.

\section{CONCLUSIONS}

A new methodology based on using video recordings for the marking of lab reports in undergraduate teaching labs has been introduced. The video corrections were performed by recording the computer's screen and the lecturer voice while marking the student reports, which are given as .pdf documents. The videos included a detailed identification and explanation of the mistakes found, and allowed the use of supporting documents (e.g. notes, internet material) to improve the explanations. The use of this methodology produces a significant reduction in the time required by the lecturer for the marking, compared to the use of annotated text in the .pdf reports. More importantly, the students warmly welcomed the corrections in this video format and all of them considered that it was completely adequate and the mistakes made were properly displayed. Moreover, $55 \%$ of them found the video corrections as one of the things they liked most from the lab sessions. Also, $90 \%$ of the students considered that the video corrections helped them to improve their report writing skills, and $70 \%$ underlined that video corrections should be generally adopted in all the subjects involving lab sessions. Finally, this methodology also served to improve the motivation of $20 \%$ of the students. All these findings demonstrate that video corrections are an excellent way to provide feedback to the students, providing a more enjoyable experience, and contributing to improve their learning process.

\section{ACKNOWLEDGEMENTS}

Financial support is acknowledged from the Universitat Jaume I under the GIE project "Evaluation of continuous evaluation tasks using video corrections" (code 3571/18). The assistance of José Ortega and Raquel Oliver is also acknowledged. 


\section{REFERENCES}

[1] F. Romero-Gavilán and J. García-Cañadas, "Flipped Classroom Evaluation Using Kahoot and Moodle in the Undergraduate Teaching Lab," in Proceedings of EDULEARN18 Conference, 2018, pp. 1985-1990.

[2] J. R. Brigati and J. M. Swann, "Facilitating Improvements in Laboratory Report Writing Skills with Less Grading: A Laboratory Report Peer-Review Process," J. Microbiol. Biol. Educ., vol. 16, no. 1, pp. 61-68, 2015.

[3] T. Hicks, J. Bruner, and T. Kaya, "Implementation of Blogging as an Alternative to the Lab Report," Int. J. Eng. Educ., vol. 33, no. 4, pp. 1257-1270, 2017.

[4] "Screencastify." [Online]. Available: https://www.screencastify.com/. [Accessed: 20-Jan-2019]. 